This is a postprint version of the following published document:

Antonelli, C., Serrano, B., Baselga, J. \&

Cabanelas, J. C. (2014). Fluorescence probes the early formation of network at the interface of epoxy-silica nanocomposite during curing. Materials Letters, 137, pp. 460-463.

DOI: 10.1016/j.matlet.2014.09.070

(C) Elsevier, 2014

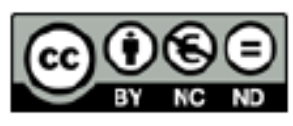

This work is licensed under a Creative Commons Attribution-NonCommercialNoDerivatives 4.0 International License. 


\title{
Fluorescence probes the early formation of network at the interface of epoxy-silica nanocomposite during curing
}

\author{
Claire Antonelli, Berna Serrano, Juan Baselga, Juan Carlos Cabanelas* \\ Department of Materials Science and Engineering and Chemical Engineering, Universidad Carlos III de Madrid, Avda. Universidad, 30, 28911, Leganés, Madrid, Spain
}

Corresponding author. E-mail address: caba@ing.uc3m.es (J.C. Cabanelas).

\begin{abstract}
The effect of surface modified silica on the cure behavior at the interface of epoxy-silica nanocomposites has been analyzed monitoring the fluorescence of the dansyl probe located at different distances from the silica surface by means of molecular tethers. FTIR analysis revealed the catalytic role of the surface hydroxyls, which is modulated by the surface modification. Fluorescence results show that network formation and associated phenomena as gelation and vitrification occurs first at the interface. The exchange of branched species throughout the permeable interface spreads its influence to the whole system until vitrification is reached.
\end{abstract}

\author{
KEYWORDS \\ Epoxy nanocomposites \\ Network formation \\ Interface \\ Fluorescence monitoring
}

\section{Introduction}

The knowledge of the network structure and topology are fundamental aspects to understand the properties of thermoset nanocomposites. Nanofillers may induce network disruption [1], and cause local off-stoichiometric compositions, which generate a heterogeneous distribution of crosslinking densities [1-6] that modify both the mechanical properties $[2,3,5]$ and the $\operatorname{Tg}[3,4]$. In addition, surface hydroxyl groups may catalyze the curing in epoxies although surface functionalization of the nanofillers inhibit this effect [7-10]. Differential segregation, network disrup-tion and catalysis obviously depend on the surface chemistry of the nanofiller and, as a consequence, the network formation must be different at the interface respect to the bulk.

Conventional kinetic models have been extensively applied to study the curing of these nanocomposites, despite the well-known strong perturbation induced by the nanofillers, which may modify the final conversion $[6,11]$, the kinetics of the curing process [6-9,11], and the occurrence of the vitrification phenomena [7,9-11]. New models are therefore needed to evaluate the heterogeneous network formation associated to the presence of nanofillers.

This work analyzes the dynamics of network formation at the interface in epoxy/silica nanocomposites by means of fluores-cence. A solvatochromic probe, dansyl chloride (DNS), has been attached to the silica surface using tethers of different length, based on organosiloxanes (short tethers) and Jeffamines (long tethers), allowing thereby to locate the chromophore at a variable distance from the silica surface. Fluorescence emission of DNS is highly sensible to its local environment [12-14] making possible to follow the cure reaction at a molecular scale. The local information obtained by fluorescence will be compared with the bulk information obtained by infrared spectroscopy, revealing a faster network formation at the interface and allowing the determination of an interface apparent epoxy conversion.

\section{Experimental section}

Colloidal suspension of silica nanoparticles (mean average size by SEM of $13 \pm 5 \mathrm{~nm}$ ), in $N, N$-dimethylacetamide, DMAC-ST, was supplied by Nissan Chemical Industries Ltd. Short tether modifiers used were (3-aminopropyl)dimethylethoxysilane (APDMES) and (3-glycidoxypropyl)dimethylethoxysilane (GPDMES), both from ABCR GmbH. Large tethers, O,O'-bis(2-aminopropyl)polypropylene glycol (Jeffamine D-230 and D-400, $M_{\mathrm{w}}$ ca. 230 and $400 \mathrm{~g} / \mathrm{mol}$ ) were supplied by Fluka. Two dansyl derivatives were used, 5dimethylaminonaphthalene-1-sulfonyl chloride (dansyl chloride) for APDMES and Jeffamines labeling, and $N$-(dimethylaminonaphthalenesulfonyl)-1,5-pentanediamine for GPDMES labeling, supplied by Invitrogen and Fluka respectively. Matrix precursors were poly(bisphenol A-co-epichlorohydrin), glycidyl end-capped (DGEBA, $\quad M_{\mathrm{w}}=348 \mathrm{~g} / \mathrm{mol}$ ) and $m$-xylylenediamine (MXDA, $\left.M_{\mathrm{w}}=136.19 \mathrm{~g} / \mathrm{mol}\right)$, supplied by Sigma Aldrich.

Surface modifiers were first labeled by DNS. The structure and length of the molecular tethers and the modifier:DNS molar ratio are given in Table 1 . Silica was directly functionalized by the 
Table 1

Structure and length of silica chemical modifiers.

\begin{tabular}{|c|c|c|c|}
\hline Labeled tether structure & Tether:DNS molar ratio & Tether length ${ }^{a}$ & Nanocomposite designation \\
\hline & $40: 1$ & 5 & $\mathrm{~N} 2 \mathrm{AD}$ \\
\hline & $40: 1$ & 15 & N2GDC \\
\hline & $40: 10: 1^{b}$ & 21 & $\mathrm{~N} 2 \mathrm{~J} 230(n=2.5)$ \\
\hline & $40: 10: 1^{b}$ & 35 & $\mathrm{~N} 2 \mathrm{~J} 400(n=6.1)$ \\
\hline
\end{tabular}

labeled tethers except in the case of Jeffamines, which were grafted onto silica previously silanized with GPDMES. Further details can be found in the supplementary data.

Suspensions of modified silica were added to DGEBA in DMAC solution under strong agitation, in order to achieve $2 \%$ by weight of silica in the nanocomposite. After vacuum solvent removal, a stoichiometric amount of MXDA was added and the mixture was cured at $80{ }^{\circ} \mathrm{C}$ for $90 \mathrm{~min}$. Nanocomposite designations are presented in Table 1 . Two reference samples have been also prepared labeling the hardener MXDA with dansyl to locate the chromophore in the bulk. Those were neat epoxy and the nanocomposite prepared with untreated silica (designed N2D).

Fourier transform near infrared spectroscopy (FTniR, PerkinElmer GX2000) was used to determine the epoxy conversion during the cure. Reaction mixtures were placed in a thermostatic cell and the signal of epoxy groups at $\sim 4526 \mathrm{~cm}^{-1}$ was followed to calculate the conversion [15]. Cure monitoring by fluorescence steady-state spectroscopy was performed with a FS900CDT fluorimeter (Edinburgh instruments) coupled to a Perkin-Elmer DSC7 calorimeter oven by a bifurcated optical light guide. The spectra were obtained between $368 \mathrm{~nm}$ and $670 \mathrm{~nm}$. Excitation was set at $350 \mathrm{~nm}$.

\section{Results and discussion}

Fig. 1shows the conversion profiles for the neat epoxy and the nanocomposites. In all cases, reaction becomes diffusion controlled above 20-25 min, reaching a common final conversion around 0.90 in the vitrified systems. The acceleration of the cure induced by the silanol groups $[7,10]$ is clearly observed. Silica surface treatment reduces both silanols concentration and activity due to the steric hindrance introduced by the modifiers. Notwithstanding, their contribution to the network formation is difficult to evaluate as they are immobilized and concentrated on the silica surface, contrary to DGEBA hydroxyls which are available throughout the reaction volume.

Fluorescence allows to study the network developing in the vicinity of the silica surface. The change of the first moment of the fluorescence emission in wavenumber units, $\nu$, which is defined as $\nu=\int I_{R}(\nu) \nu d \nu / \int I_{R}(\nu) d \nu$, where $I_{\mathbb{R}}(\nu)=I_{\mathbb{R}}(\lambda) \lambda^{2}[16]$ was used for cure

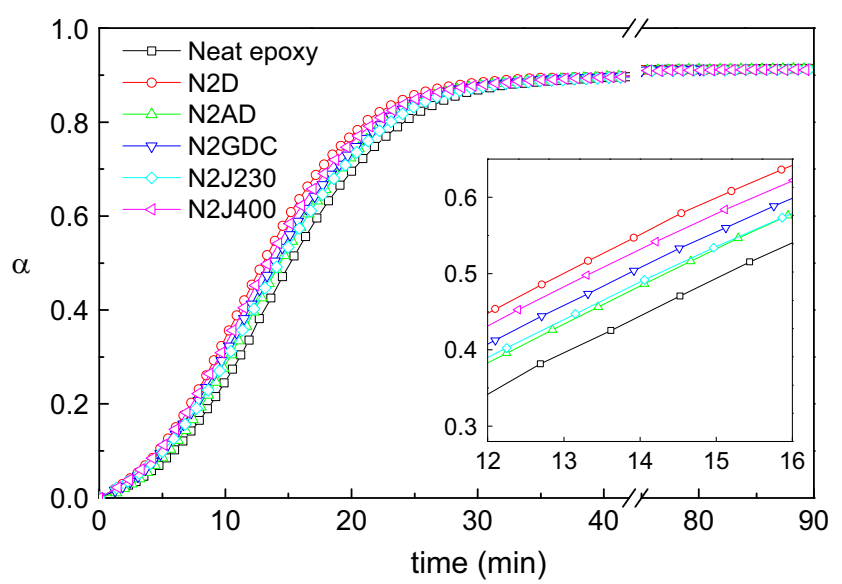

Fig. 1. Epoxy conversion by FTnIR, $\alpha$, against curing time at $80^{\circ} \mathrm{C}$ for the neat epoxy and nanocomposites.

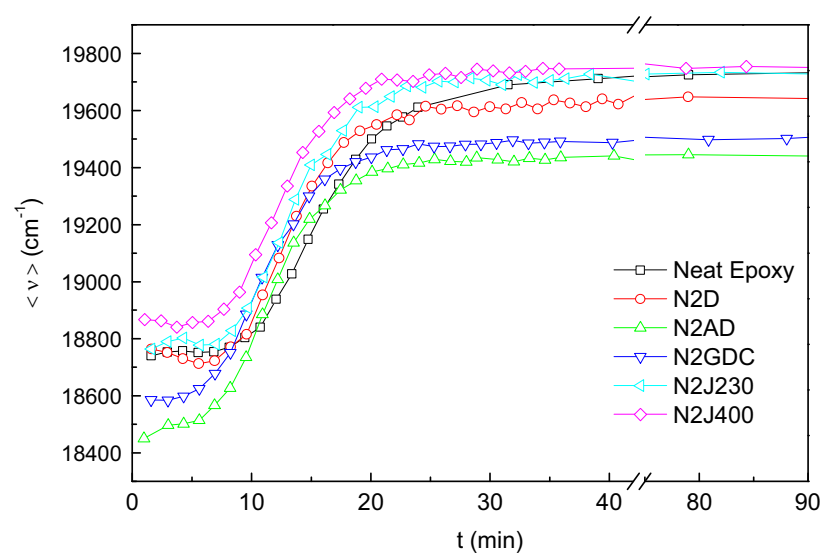

Fig. 2. First moment of dansyl emission fluorescence, $\langle v\rangle$, against curing time at $80{ }^{\circ} \mathrm{C}$ for the neat epoxy and nanocomposites.

monitoring. The $\nu$ parameter is related with the mean energy of emitted photons, and is very sensitive to changes in the chromophore environment [17]. Fig. 2shows $\nu$ against the curing time. 


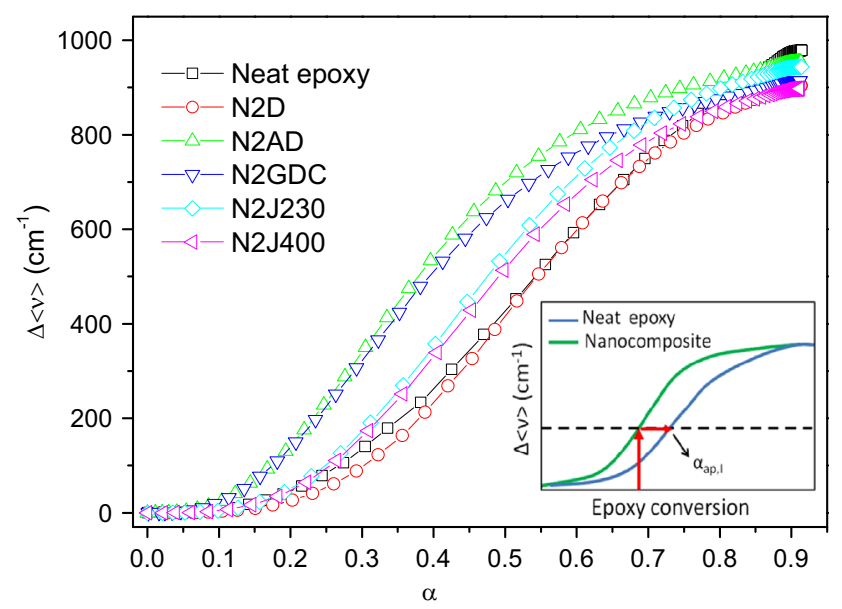

Fig. 3. $\Delta\langle v\rangle$ against epoxy conversion, $\alpha$, for the neat epoxy and nanocomposites.

A spectral blue shift or, equivalently, an increase of $\nu$, is observed in all samples. The blue shift is caused by the increase of medium rigidity as curing proceeds, which hampers the stabilization and relaxation of the highly polar excited state by the surrounding dipoles [18]. Above 20 min $\nu$ reaches a maximum constant value, which coincides with the vitrification of the system.

The small variations observed in the initial value of $\nu$ are due to the different local polarity. A lower value of $\nu(0)$ is related with the presence of surface silanols, providing a more polar environment which promotes excited-state relaxation. Therefore, neat epoxy and N2D samples, with DNS located in the bulk, have a similar $\nu(0)$ value, while N2AD and N2GDC samples, where DNS is located very close to the silica, present a lower $\nu(0)$ value.

Surprisingly, when samples reach vitrification the absolute increment of $\nu$, defined as $\Delta \nu=\nu(\infty)-(0)$, is comparable for all the samples, in the order of $900 \mathrm{~cm}^{-1}$ (about $0.1 \mathrm{eV}$ ). This behavior correlates well with the common final macroscopic conversion. Therefore, the observed $\Delta \nu$ is associated to the rigidity increase with curing. In order to explore the curing at different DNS locations, the difference $\Delta \nu(t)=\nu(t)-\nu(0)$ was plotted against epoxy conversion determined by FTnIR, as shown in Fig. 3. Notable differences in $\Delta \nu(t)$ were observed depending on DNS location. When dansyl is close to the silica surface (N2AD and N2GDC), $\Delta \nu(t)$ begins to increase before and grows faster than in neat epoxy. At epoxy conversions around $0.6, \Delta \nu(t)$ values begin to converge until a similar value at high conversions is reached. For DNS attached to long tethers (Jeffamines), the behavior is intermediate, as expected, because dansyl must be more embedded in the bulk. Thus, the fluorescence response of dansyl permits to monitoring in real time the catalytic role of surface silanols and shows clearly the earlier development of the network at the interface.

It is possible to estimate an apparent conversion at the interface, $\alpha_{\mathrm{ap}, \mathrm{I}}$, comparing $\Delta \nu(t)$ of the neat epoxy, where the chromophore is homogeneously distributed, with $\Delta \nu(t)$ of the nanocomposite. The method is depicted in the inset of Fig. 3; similar $\Delta \nu(t)$ implies a similar rigidity of the local environment of the chromophore and, thus, similar network structure. Conversion data obtained using this method must be considered apparent because the chemical composition at the interface may be slightly different from the bulk but the compositional changes on the surroundings of DNS (Fig. 2) are responsible of minor differences in energy emission compared to those produced by the curing; in addition, these initial differences in $\nu$ are roughly maintained after the curing and therefore its influence in $\Delta \nu$ is very low. Fig. 4 presents the values of $\alpha_{\mathrm{ap}, \mathrm{I}}$, confronted with the global epoxy conversion. The conversion obtained at the interface when dansyl is very close to the silica surface (N2AD and N2GDC) is always higher than the global

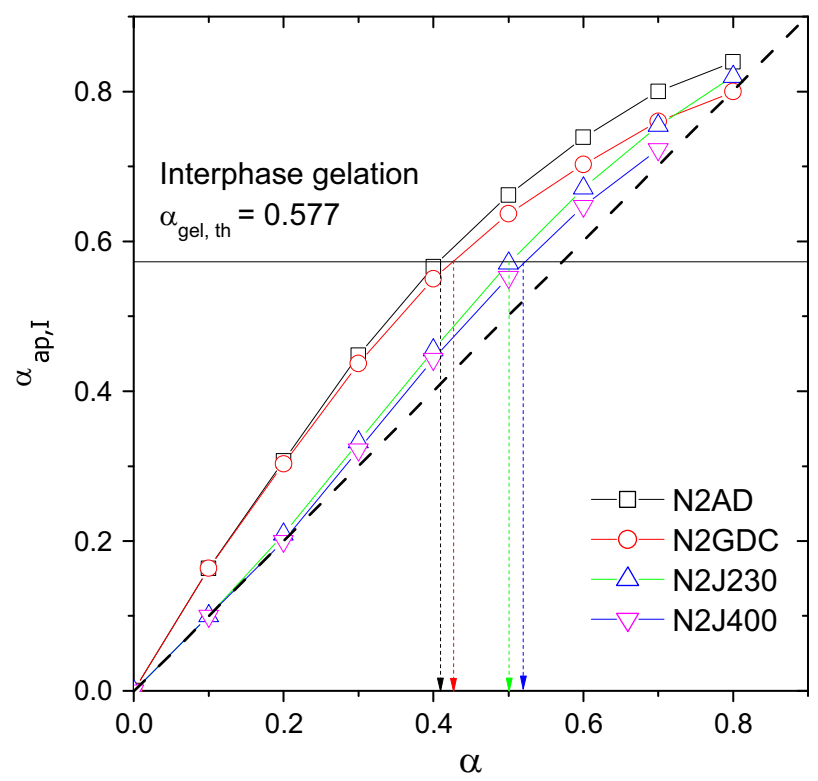

Fig. 4. Estimated conversion at the interphase, $\alpha_{\mathrm{ap}, \mathrm{l}}$, against epoxy conversion, $\alpha$, for the nanocomposites. Gel conversion was calculated theoretically [19]. Vertical arrows indicate the macroscopic conversion at the interphase gelation.

conversion showing the effect of the surface hydroxyls at the earlier stages of the curing, responsible of the high differences between the bulk and the interface. The maximum observed difference is about $15 \%$ at the intermediate stage of the curing suggesting that gelation, which is scarcely influenced by small changes in the stoichiometry [19], is reached earlier at the interface.

At the beginning of the reaction, the interface is permeable and the exchange of branched species with the bulk is allowed, with a net transfer of high molecular weight species from the interface to the bulk. This fact translates the acceleration effect of the silanol catalysis to the whole sample. When the apparent conversion at the interface is high, well above gel point, diffusion control becomes important and progressively both conversions converge. Therefore, at high conversions at the interface the influence of silanols catalysis decays even though the whole system is far away from vitrification.

For nanocomposites modified with Jeffamines, the variations of $\alpha_{\mathrm{ap}, \mathrm{I}}$ with neat epoxy are much less pronounced and appear later. When DNS is more embedded in the bulk, the interface network formation is detected later and the influence of silica surface is lower. This observation suggests that interface thickness is very small, at most $3 \mathrm{~nm}$ according to tether lengths, since the chromophore must be in a distribution of sites around the nanoparticles depending on the molecular conformation of the tether. It also evidences a gradient growth of the network from the surface to the bulk.

\section{Conclusions}

Fluorescence monitoring of the curing reaction at the interface revealed that conversion at the interface is always a step ahead of the bulk conversion, from up to $15 \%$. A permeable interface at the beginning of the reaction allows an efficient exchange of branched species with the bulk thus accelerating the curing over a broad epoxy conversion range. This study unambiguously shows that network formation and associated phenomena as gelation and vitrification occurs first at the interface, with a probable repercussion on the rheology during curing and on the resulting network morphology and final properties of the nanocomposite. 
These findings should be considered in any kinetic modelling of the curing process of epoxy nanocomposites.

Supplementary data: Supplementary data about sample preparation can be found at http://www.sciencedirect.com.

\section{Acknowledgements}

Authors are gratefully acknowledged to Spanish Ministerio de Economía y Competitividad for financial support under grant MAT2010-17091.

\section{References}

[1] Preghenella M, Pegoretti A, Migliaresi C. Thermo-mechanical characterization of fumed silica-epoxy nanocomposites. Polymer 2005;46:12065-72.

[2] Chen C, Justice RS, Schaefer DW, Baur JW. Highly dispersed nanosilica-epoxy resins with enhanced mechanical properties. Polymer 2008;49:3805-15.

[3] Zaman I, Phan TT, Kuan HC, Meng Q, Bao La LT, Luong L, et al. Epoxy/graphene platelets nanocomposites with two levels of interface strength. Polymer 2011;52:1603-11.

[4] Putz KW, Palmeri MJ, Cohn RB, Andrews R, Brinson LC. Effect of cross-link density on interphase creation in polymer nanocomposites. Macromolecules 2008;41:6752-6.

[5] Zhang H, Zhang Z, Friedrich K, Eger C. Property improvements of in situ epoxy nanocomposites with reduced interparticle distance at high nanosilica content. Acta Mater 2006;54:1833-42.

[6] Rosso P, Ye L. Epoxy/silica nanocomposites: nanoparticle-induced cure kinetics and microstructure. Macromol Rapid Commun 2007;28:121-6.

[7] Sanctuary R, Baller J, Zielinski B, Becker N, Krüger JK, Philipp M, et al. Influence of $\mathrm{Al}(2) \mathrm{O}(3)$ nanoparticles on the isothermal cure of an epoxy resin. J Phys Condens Matter Inst Phys J 2009;21:035118-1-8.

[8] Ryu SH, Sin JH, Shanmugharaj aM. Study on the effect of hexamethylene diamine functionalized graphene oxide on the curing kinetics of epoxy nanocomposites. Eur Polym J 2014;52:88-97.

[9] Zhou T, Gu M, Jin Y, Wang J. Effects of nano-sized carborundum particles and amino silane coupling agent on the cure reaction kinetics of DGEBA/EMI-2,4 system. Polymer 2005;46:6216-25.

[10] Alzina C, Sbirrazzuoli N, Mija A, Epoxy-Amine Based Nanocomposites. Reinforced by silica nanoparticles. Relationships between morphologic aspects, cure kinetics, and thermal properties. J Phys Chem C 2011;115:22789-95.

[11] Baller J, Becker N, Ziehmer M, Thomassey M, Zielinski B, Müller U, et al. Interactions between silica nanoparticles and an epoxy resin before and during network formation. Polymer 2009;50:3211-9.

[12] Wang Jeffrey Z, Song JC, Bao R, Neckert DC. Fluorescence probes for monitoring polymerization processes. J Polym Sci Part B Polym Phys 1996;34:325-33.

[13] Vatanparast R, Li S, Hakala K, Lemmetyinen H. Monitoring of curing of polyurethane polymers with fluorescence method. Macromolecules 2000;33:438-43.

[14] Baselga J, Piérola IF, Serrano B, Pozuelo J, Cabanelas JC, Martín O. Fluorescence of polymers at interfaces: polymerization, relaxations, and imaging. Rev Fluoresc 2010;2010 (311-47).

[15] Mijovic J, Andjelic S. A Study of Reaction Kinetics by Near-Infrared Spectroscopy1. Comprehensive analysis of a model epoxy/amine system. Macromolecules 1995;28 (2797-806).

[16] Valeur B. Molecular fluorescence: principles and applications. first ed.. Weinheim: Wiley-VCH; 2001

[17] Mikes F, Gonzalez-Benito F, Serrano B, Bravo J, Baselga J. Fluorescence monitoring of polymerization reaction. A new method for treating fluorescence experimental data. Polymer 2002;43:4331-9.

[18] Jager WF, Volkers AA, Neckers DC. Solvatochromic fluorescent probes for monitoring the photopolymerization of dimethacrylates. Macromolecules 1995;28:8153-8.

[19] Pascault JP, Sautereau H, Verdu J, Williams RJJ. Thermosetting. Polymers. first ed.. New York: Marcel Dekker; 2002. 